\title{
Research of Neuron Growth Prediction and Influence of Its Geometric Configuration*
}

\author{
Tao Sun, Liang Lin, Qiaoyu Huang \\ College of Science, Guilin University of Technology, Guilin, China \\ E-mail:Lzcst135@163.com,Linliang6666@126.com,89670447@qq.com \\ Received May 6, 2011; revised May 25, 2011; accepted May 28, 2011
}

\begin{abstract}
The neuron growth will bring series variation to the neuron characteristics of geometric configuration. Especially the growth of dendrite and axon can obviously change the space characteristic and geometric characteristic of neuron. This article is to build the prediction model of neuron growth through knowing the statistics rules of neuron geometric characteristics, better imitate the neuron growth, and clearly analyze the growth influence of geometric configuration.
\end{abstract}

Keywords: Neuron, Growth Prediction, Geometric Configuration

\section{Introduction}

Neuron space geometric configuration research of is one important project of Human Brain Project (HBP). As the basic brain unit, there have many elements in neuron structure and function. Neuron geometric configuration characteristic and electric physical property are the two importance. Electrical characteristics include different potential issue mode of neuron. Geometric characteristics involve neuron space conformation, specifically dendrites to receive information, somas to manage information and axons to come out information. With regard to the hot research of geometric characteristic, different experts place extra emphasis on different index to describe the morphological characteristic of neurons. In the Research of Neuron Typological Classification and Questions Identification [1], we have classified the relatively stable neuron at normal intervals through geometric configuration characteristics and got the better effects. However, the dendrite and axon growth of neurons are various with the passes of time. This article will point at the neuron growth prediction and analyze these morphological changes to ensure the geometric characteristics effects.

\section{Neuron Growth Prediction}

The variation of neuron growth reflects in the soma area and axon length. Moreover, it is also the growth process

\footnotetext{
"Supported by the Innovation Project of Guangxi Graduate Education (Grant No. 2011105960202M31)
}

from one soma to the tree dendrite gradually. The data information provide from Neuronmorpho.org [2] do not arrange follow the time regularity. However, the present data is only the single neuron form without the growth process record. In addition, if we search for the growth regulations that we need to start from various neuron morphological characteristics. Especially analyze from the given microcosmic statistic of each room and bifurcation such as length, local branch angle, local dip angle and other geometric characteristics.

As we know, there will have a new bifurcation to growth with once neuron growth. The local branch angle, local dip angle and other geometric characteristics of different types of neurons, there need to satisfy the determinate statistics regulations. If we can find the distributed regularity about the neuron crotch, it will be possible to take the advantage of stochastic simulation to create the correct regulation of growth direction, length and so on. Imitate the entire growth process of neuron growth to reach the variety prediction of neuron modal.

Here we not only point at regulation abstraction of neuron growth but also research the physiological process of neuron structure, then to do the mechanism-based study of neuron structure.

\subsection{Theory Research}

In the conference [3], based on test description, take the hypothalamus neuron of rat, and continuously observe the change regulation of neuron somas diameter, axon 
length and numbers. Through the observation in the culture solution, we can find the dispersed hypothalamus neurons adherences in 3 hours with the shape of roundness or egg. After 12 hours, the cells aggregate to cluster and disperse growth at the same time. After 24 hours, the cells will change into triangle or polygon with special soma enation. After 36 hours growth, the shape become clearly with enation. After 48 hours, the visible soma can send out several tiny dendrites. After 3 days, the growth speed of dendrite increase every day. After 6 days, there will have thick axon connection among soma clusters with clear-cut neuron. Moreover, the soma is fullness with roundness or fusiform shape. On the seventh day, the growth of neuron soma and dendrite length will reach the peak value. On the tenth day, the vacuoles will appear in the soma and the dendrite is shrinking. Experiments shows, cultivate rat hypothalamus $(\mathrm{CRH})$ growth the most fast from the third day to the sixth day. During the seventh day to the tenth day, the neuron number, soma diameter, dendrite length and $\mathrm{CRH}$ coloration strength reach the peak. After the twelfth day, part neuron appears back change, the somas become shrinking, the rounding glow is fading, the number is reducing and the coloration is weakening.

From the above data, we can predict the growth variation of neuron. During the growth process, the growth rate from slow to fast and then gradually slow to stop. Moreover, the neuron has the process from small to large then to the last shrink. The predict growth curve is Figure 1.

\subsection{Growth Imitation Prediction}

In order to achieve the computer imitation of neuron growth, we can discrete the growth time. During each section of growth period, all the termination point will appear fixed rate growth. The offspring of termination could be one or two then will appear the branch growth. This kind of growth occurrence time suppose as the

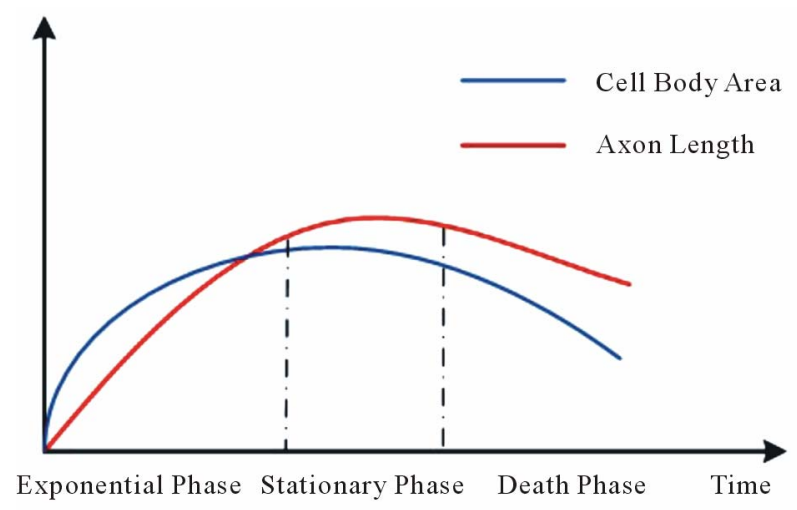

Figure 1. Growth cycle curve of neuron. primary time of growth period. While in the computer imitation, the growth can also predict as:

- In the system, only consider each level growth of branch direction, diverge and length bout the motor neuron but ignore the dendrite diameter, soma diameter change during the growth, there things cannot be ignored while analyzing.

- The characteristics of neuron growth direction and length need to satisfy the normality distribute random.

- The neurons always grow in the imitation period without limitation.

- In once growth, the characteristic that has room will not change (length, direction). Moreover, the external surface rooms grow the filial generation room.

Here we chose the neuron with big soma, huge trunk and similar to the ball growth. It only has dendrite without axon characteristics as reference. Next will introduce the prediction implement through prediction model of neuron growth.

The process description is:

There has only soma in the initial growth. The soma is a ball, the branch has to grow from the cell and the branch number is the random number of obeyed normality distribution $N_{1}$. Branch length is one obeyed normality distribution $N_{2}$. The direction is random; the most external surface room has probability $p$ to growth the new room. Moreover, the branch probability is $q$. The most external surface room and part dip angle of newborn room is the obeyed normality distribution $N_{3}$. If it is branch, the first newborn room and the second angle is the random number of obeyed normality distribution $N_{4}$. The growth number of times is Num. The Process model of neuron growth prediction is Figure 2.

- Get one random number of obeyed $N_{1}$ is the number of gronw room. The length abstract one obeyed normality distribution $N$ of random number and grows level one room.

- $k=2,3,4, \cdots$ Judge whether $k$ can reach the growth number or not. The time is Num. If reach the number, stop doing that.

- Judge whether $k$ level room can grow the room or not $(k=2,3,4, \cdots)$ If grow, jump to 4 meters, if not, $k=k+1$ then to 2 .

- Judge the $k$ level room is branch or not: if not, jump to 5 . If it is, jump to 6 .

- The part dip angle of $k+1$ level room and $k$ level room chose one random number to obey $N_{3}$, the length can chose one random number to obey $N_{2}$, and it should grow $k+1$ level room. $k=k+1$, jump to 2 .

- The part dip angle from first level of $k+1$ room and $k$ level room that can chose one random number to obey $N_{3}$, the length from the random number to obey $N_{2}$ then to grow the first room of $k+1$ level. 
- The part branch angle of second room and the first room is a random number to obey $N_{4}$. The length is a random number to obey $N_{2}$. When grow the second room of $k+1$ level, jump to 2 .

Note 1: if it is branch, the part dip angle of first growth room is less than the second growth room.

Note 2: 2-7 steps is the growth process of $k$ level room, all the $k$ level rooms are same as this.

Note 3: the incidental system of this article, $N_{1}$ normality distribution average and variance is all the neuron branch average and variance between A and B. the normality distribution average of $N_{2}, N_{3}$ and $N_{4}$ is the average and variance of neuron room length, part dip angle and part branch angle.

The system imitates the neuron growth and the system operation get three imitation growth neurons, such as Figures 3-5 shows:

At the closer soma part, the dendrite growth gets better imitation.

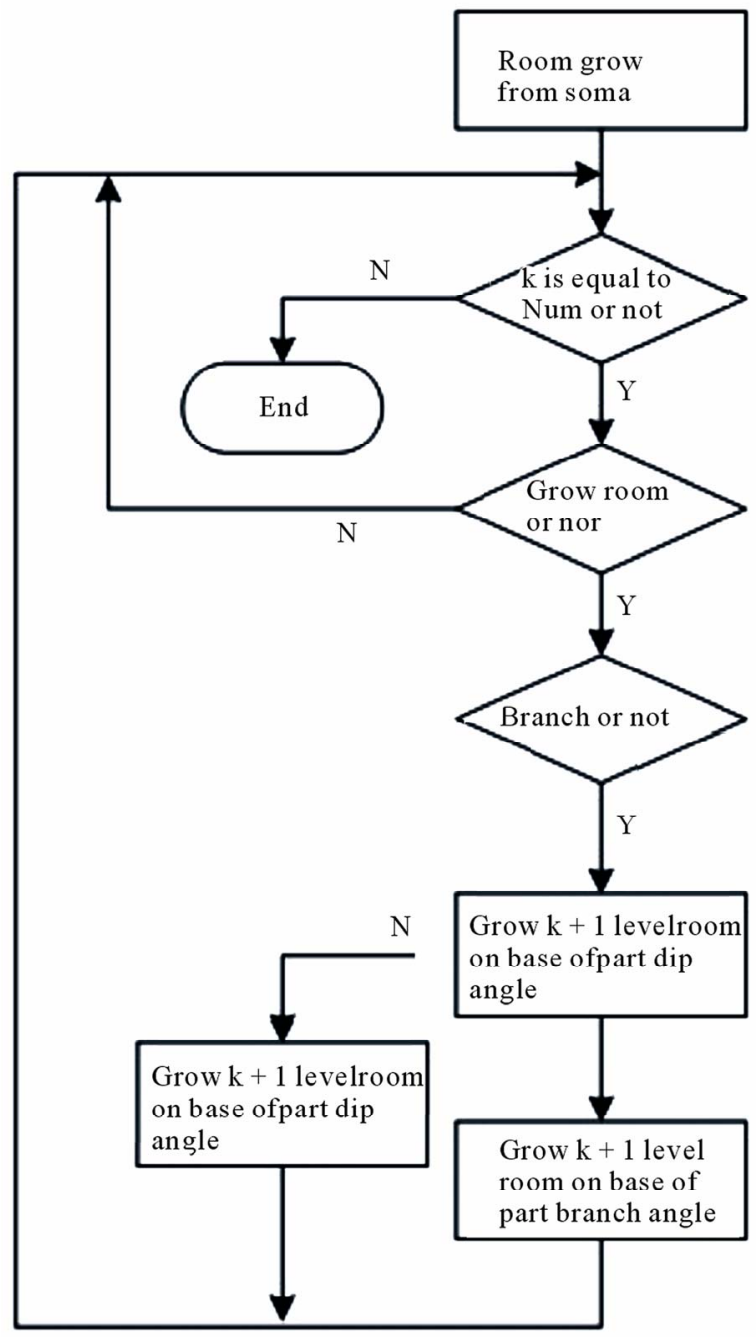

Figure 2. The Process model of neuron growth prediction.

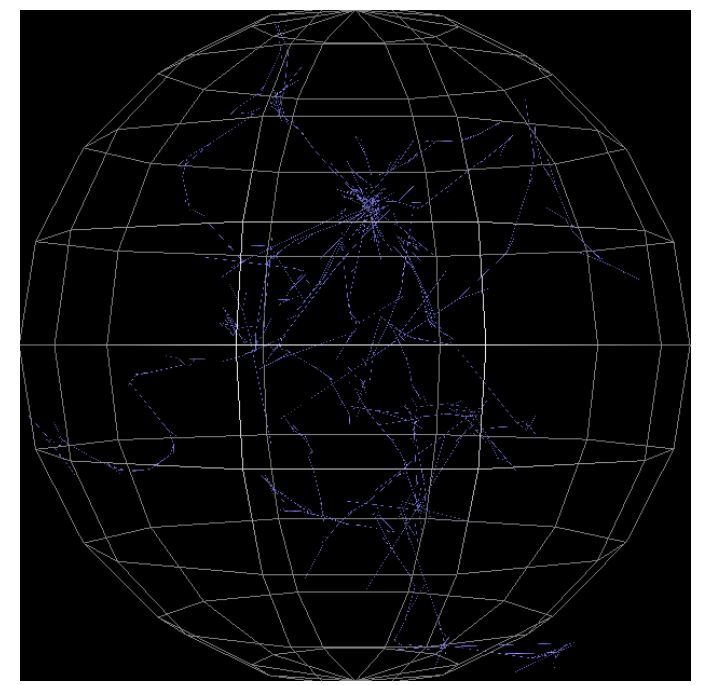

Figure 3. Neuron growth imitation 1.

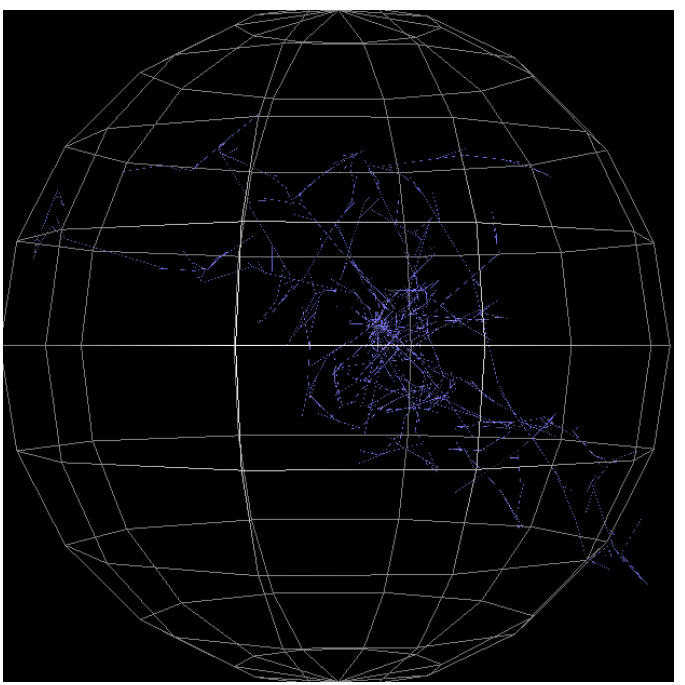

Figure 4. Neuron growth imitation 2.

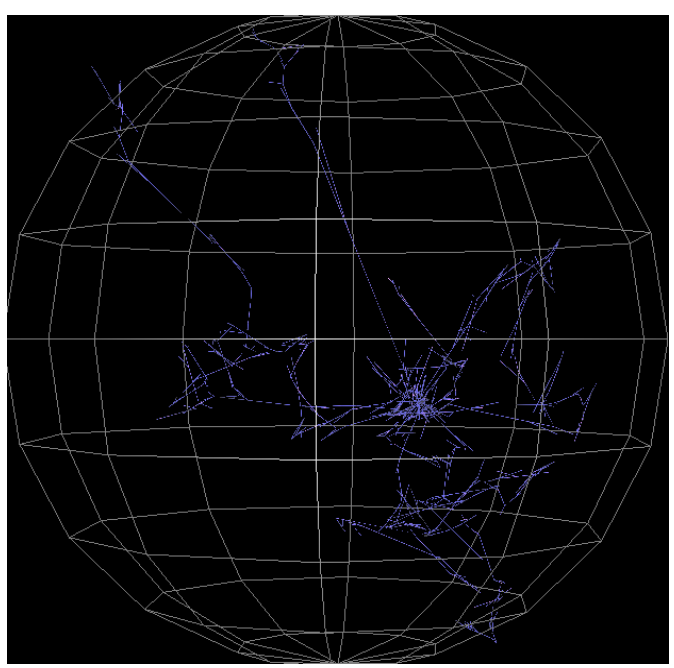

Figure 5. Neuron growth imitation 3. 
Not all the crazy growth of trees is suit with the neuron growth regulations. This is created by the suppose 4 . In the growth of real neuron, each tree will not be limited until reach the certain degree. The most external surface growth is random so this is the most important reason.

The length of some rooms is too long. If set one threshold to chose from the obeyed random number of normality distribution, it can avoid this unmoral condition. Nevertheless, the threshold cannot determine that not set this subject.

\section{Neuron Growth Prediction and Its Influence of Geometric Characteristics}

According to the research of neuron morphologic, different function of neuron has huge differences in size, shape and complexity [4]. The axon is long in common. It is separate from axon hillock with uniformity diameter. The commence part is the begin part. Apart from the soma some distances, we will get the myelin sheath, which called nerve fiber. The soma differences are huge. The small diameter is only 5-6 $\mu \mathrm{m}$ and the large one could be even $100 \mu \mathrm{m}$ and more. The dendrite form, number and length are different. Dendrite most be branch, it can transform to the somas after stimulation. Axons are funicular. The terminal have branches and called axon terminal. Axons transform from soma to the terminal. Usually, one neuron has one or more dendrites. Nevertheless, axon has only one. The bigger neuron is the longer the axon is. Now divide the neuron growth process into exponential phase, stationary phase and death phase. Target is as the length of neuron dendrite. Separately describe the growth change and impact of geometric characteristic in different phases. Here we predict the following things:

Exponential phase: The half diameter is small. Nevertheless, it will grow more branches with obviously changes. With the increase of the diameter, the broadcast consume of neuron signal will increase all the same. At the same time, the metabolic costs also increase the same. The change of form will influence the geometrical form and it might have mistakes.

Stationary phase: the space form of neuron is ensuring. Under the rich growth, there will not have a change in a period [5]. However, on the limited environment, the neuron will limit the growth even grow back. So the ensure form data should reflects the geometric form. It is the best periods of neuron form and functional predic- tion.

Death phase: when the neuron and neighboring somas is same to the certain extent, the neuron growth will be limited. Some will back growth and the whole neuron structure will change more and not suit for the neuron identification and classification.

\section{Conclusions}

Neuron growth will bring the series change of geometrical characteristics. Especially the growth of dendrite and axon will change the space characteristic and geometric characteristic obviously. This article is through the identification of spastics regulations and builds the prediction model. The growth prediction of combine neuron structure knowledge, take the advantage of growth period and describe the form structure and function such as growth, death, branch and so on. Use the rate distribution and random process theory to analyze the neuron topological structure regulation. Through the model identification and abstract the growth regulation. The model applies Monte Carlo to imitate the neuron growth. For the close soma part, dendrite growths get the better imitation. However, far side room growth cannot control reasonable. There has no whole reflect condition of neuron growth. If want to do the further research, the initial thinking is increasing with the growth configuration and reduction.

\section{References}

[1] T. Sun, L. Lin and Q. Y. Huang, "Research of Neuron Typological Classification and Identification Questions," (Committed)

[2] NeuroMorpho.Org. 2010. http://neuromorpho.org/neuroMorpho/index.jsp

[3] Y. D. Zhang, P. F. Zhu and Z. G. Wang, "Research of Neuron Regulation about Cultivate Rat Hypothalamus," Journal of Traumatic Surgery, Vol. 6, No. 1, 2004, pp. 45-47.

[4] M. B. Kerry, "Quantifying Neuronal Size: Summing up Trees and Splitting the Branch Difference," Seminars in Cell \& Developmental Biology, Vol. 19, No. 6, 2008, pp. 485-493. doi:10.1016/j.semcdb.2008.08.005

[5] G. A. Ascoli, et al., "Generation, Description and Storage of Dendritic Morphology Data," Philosophical Transactions of the Royal Society B, Vol. 356, No. 1412, 2001, pp. 1131-1145. doi:10.1098/rstb.2001.0905 